\title{
Stochastic processes crossing from ballistic to fractional diffusion with memory: exact results
}

\author{
V. Ilyin'1, I. Procaccia ${ }^{1}$, A. Zagorodny ${ }^{2}$ ] \\ 1 Department of Chemical Physics, The Weizmann Institute of Science, Rehovot 76100, Israel \\ 2 Bogolyubov Institute for Theoretical Physics, 252143 Kiev, Ukraine
}

Received March 15, 2010

\begin{abstract}
We address the now classical problem of a diffusion process that crosses over from a ballistic behavior at short times to a fractional diffusion (sub- or super-diffusion) at longer times. Using the standard non-Markovian diffusion equation we demonstrate how to choose the memory kernel to exactly respect the two different asymptotics of the diffusion process. Having done so we solve for the probability distribution function as a continuous function which evolves inside a ballistically expanding domain. This general solution agrees for long times with the probability distribution function obtained within the continuous random walk approach but it is much superior to this solution at shorter times where the effect of the ballistic regime is crucial.
\end{abstract}

PACS: $05.10 . \mathrm{Cg}, 05.20 . \mathrm{Dd}, 51.10+y$

Key words: fractional diffusion, memory effects, ballistic processes

\section{Introduction}

Since the classical work of Hurst [1] on the stochastic discharge of reservoirs and rivers, Nature has offered us a large number of examples of diffusion processes which are 'anomalous' in the sense that an observable $X$ diffuses in time such that its variance grows according to

$$
\left\langle\Delta X^{2}\right\rangle(t) \sim D_{\alpha} t^{\alpha} \text { for } t \gg t_{\mathrm{c}},
$$

where $\alpha \neq 1$, angular brackets mean an average over repeated experiments and $D_{\alpha}$ is a coefficient with the appropriate dimensionality. This long time behavior with $\alpha \neq 1$ is generic when the diffusion steps are correlated, with persistence for $\alpha>1$ and anti-persistence for $\alpha<1$ [2]. The list of systems displaying such behavior is extensive and growing. Some subdiffusive examples: charge carrier transport in amorphous semiconductors [3], NMR diffusiometry in percolative [4] and porous systems [5], reptation dynamics in polymeric systems [6], transport on fractal geometries 7], diffusion of scalar tracer in convection [8] etc. Superdiffusive examples include special domains of rotating flow [9], collective slip diffusion on solid surfaces [10], Richardson turbulent diffusion [11], bulk-surface exchange controlled dynamics in porous glasses [12], quantum optics [13] etc. This multitude of examples created an urgent need to formulate novel stochastic theories to compute the probability distribution function that is associated with anomalous diffusive processes of this type 14]. However, all these past approaches considered only the long time behaviour equation (1) and disregarded the important fact that in many cases the short time behavior is different, being

$$
\left\langle\Delta X^{2}\right\rangle(t) \sim D_{2} t^{2} \quad \text { for } \quad t \ll t_{\mathrm{c}} .
$$

This short time behavior is known as "ballistic", and is generic for a wide class of processes. In this paper we show how to find the probability distribution function of a process that satisfies equation (11) and (2) simultaneously in the asymptotic limits. The presence of (2) at short times effects the solution in a fundamental way that cannot be neglected.

The correlations between stochastic steps mean that the diffusion process is not Markovian, but rather has memory. Thus the probability distribution function of the observable $X, f(X, t)$ is 
expected to satisfy a diffusion equation with memory [15],

$$
\frac{\partial f(X, t)}{\partial t}=\int_{0}^{t} \mathrm{~d} t^{\prime} K\left(t-t^{\prime}\right) \nabla^{2} f(X, t)
$$

with $K(t)$ being the memory kernel and $\nabla^{2}$ the Laplace operator. In this Letter we study the class of processes which satisfy equations (21)-(3) with $\alpha<2$.

First of all we find an expression for the kernel $K(t)$ which is unique for a given law of meansquare-displacement. Second, we consider the kernel which contains both the ballistic contribution equation (2) and the long-time behavior (11). For this case we find an exact equation and a solution for equation (3). Lastly, a simple interpolation formula for the kernel is inserted to the exact equation which is then solved for the probability distribution function of $X$ without any need for the fractional dynamics approach [14]. Some interesting characteristics of the solution are described below.

\section{Determination of the kernel $K(t)$}

To determine the kernel in equation (3) we use a result obtained in [16]. Consider the auxiliary equation

$$
\frac{\partial P(X, t)}{\partial t}=\nabla^{2} P(X, t)
$$

Define the Laplace transform of the solution of equation (4) as

$$
\tilde{P}(X, s) \equiv \int_{0}^{t} \mathrm{~d} t \mathrm{e}^{-s t} P(X, t)
$$

it was shown in [16] that the solution of equation (3) with the same initial conditions can be written as

$$
\tilde{f}(X, s)=\frac{1}{\tilde{K}(s)} \tilde{P}\left[X, \frac{s}{\tilde{K}(s)}\right],
$$

where here and below the tilde above the symbol means the Laplace transform. The development that we propose here is to replace in equation (6) the Laplace transform $\tilde{K}(s)$ with the Laplace transform of the mean-square displacement. This is done by first realizing (by computing the variance and integrating by parts) that

$$
\frac{\partial\left\langle X^{2}\right\rangle(t)}{\partial t}=2 \int_{0}^{t} \mathrm{~d} t^{\prime} K\left(t-t^{\prime}\right)
$$

or, equivalently,

$$
\tilde{K}(s)=\frac{s^{2} \widetilde{\left\langle X^{2}\right\rangle}(s)}{2}=\frac{\partial\left\langle X^{2}\right\rangle(t)}{2 \partial t}+\frac{s}{2} \frac{\partial \widetilde{\left\langle X^{2}\right\rangle(t)}}{\partial t}-\frac{\partial\left\langle X^{2}\right\rangle(t)}{2 \partial t}
$$

The second line was written in order to find the time representation of $K(t)$ which is the inverse Laplace transform:

$$
K(t)=\frac{1}{2}\left(\delta(t) \frac{\partial}{\partial t}\left\langle X^{2}\right\rangle(t)+\frac{\partial^{2}}{\partial t^{2}}\left\langle X^{2}\right\rangle(t)\right)=\frac{1}{2} \frac{\partial}{\partial t}\left(H(t) \frac{\partial\left\langle X^{2}\right\rangle(t)}{\partial t}\right),
$$

where $H(t)$ is the Heaviside function. Obviously, using the first line of equation (8) in equation (6) the solution is entirely determined by whatever law is given for the variance, together with initial conditions. 
For ordinary diffusion the variance is defined by equation (11) with $\alpha=1$ and $t_{\mathrm{c}}=0$. It follows from equation (9) that the kernel is $K(t) \sim \delta(t)$ and equation (3) is reduced to the Markovian equation (41); this process does not possess any memory. More complicated examples are considered below.

\subsection{Example I: fractional differential equations.}

In recent literature the problem of a diffusion process which is consistent with equation (1) only for all times (i.e. $t_{\mathrm{c}}=0$ ) is investigated using the formalism of fractional differential equations (see, e.g., [14]). In this formalism equation (3) is replaced by the fractional equation

$$
\frac{\partial f(X, t)}{\partial t}=D_{\alpha}{ }_{0} \mathbf{D}_{t}^{1-\alpha} \frac{\partial^{2} f(X, t)}{\partial x^{2}}
$$

where the Rieman-Liouville operator ${ }_{0} \mathbf{D}_{t}^{1-\alpha}$ is defined by

$$
{ }_{0} \mathbf{D}_{t}^{1-\alpha} \phi(x, t)=\frac{1}{\Gamma(\alpha)} \frac{\partial}{\partial t} \int_{0}^{t} \mathrm{~d} t^{\prime} \frac{\phi\left(x, t^{\prime}\right)}{\left(t-t^{\prime}\right)^{1-\alpha}},
$$

where $\Gamma(\alpha)$ is the gamma function. It is easy to see that this equation follows from equation (3) with the kernel evaluated by equation (9) with the variance (11). We reiterate however that this equation is consistent with equation (1) for all times $t \geqslant 0$. This, of course, is a problem since this formalism cannot agree with the ballistic short time behavior which is generic in many systems.

\subsection{Example II: ballistic behavior}

For $X$ one-dimensional the solution of equation (4) with the initial condition $P(X, t=0)=$ $\delta(X)$ is given by

$$
\tilde{P}(X, s)=\frac{1}{2 \sqrt{s}} \cdot \exp (-|X| \sqrt{s}) .
$$

Substituting in equation (6) we find

$$
\tilde{f}(X, s)=\frac{1}{\sqrt{2 s^{3} \widetilde{\left\langle X^{2}\right\rangle}(s)}} \exp \left[-|X| \sqrt{\frac{2}{s \widehat{\left\langle X^{2}\right\rangle}(s)}}\right] .
$$

For systems with the pure ballistic behavior (e.g., dilute gas) the variance can be written as $\left\langle X^{2}\right\rangle_{t}=\left\langle u^{2}\right\rangle t^{2}$, where $\left\langle u^{2}\right\rangle$ is the mean square average of the particle velocities. The Laplace transform of this expression is given by $\widetilde{\left\langle X^{2}\right\rangle}(s)=2\left\langle u^{2}\right\rangle / s^{3}$ and the Laplace transform of the probability distribution function is defined by

$$
\tilde{f}(X, s)=\frac{1}{2 \sqrt{\left\langle u^{2}\right\rangle}} \exp \left(-|X| \frac{s}{\sqrt{\left\langle u^{2}\right\rangle}}\right) .
$$

The inverse transform reads

$$
f(X, t)=\frac{1}{2} \delta\left(|X|-\sqrt{\left\langle u^{2}\right\rangle} t\right) .
$$

This solution corresponds to a deterministic evolution; there is a complete memory of the initial conditions in the absence of inter-particle interactions, $\left.K(t)=\left\langle u^{2}\right\rangle\right)$.

\section{General case}

In the general case the mean-square-displacement satisfied some law $\left\langle X^{2}\right\rangle(t)$ which is supposed to be known at all times, with possible asymptotic behavior as shown in equation (2) and(1). To 
find the appropriate general solution we will split $\tilde{f}(X, s)$ into two parts, $\tilde{f}_{\mathrm{I}}(X, s)$ and $\tilde{f}_{\mathrm{II}}(X, s)$, such that the first part is constructed to agree with the existence of a ballistic regime. Suppose that in that regime, at short time, the mean-square-displacement can be expanded in a Taylor series

$$
\left\langle X^{2}\right\rangle(t)=\sum_{i=0}^{\infty} a_{i} t^{\mu_{i}-1}=a_{0} t^{2}+a_{1} t^{3}+a_{2} t^{4} \cdots,
$$

where $\mu_{0}=3, \mu_{1}=4$ etc. Then the Laplace transform $\widetilde{\left\langle X^{2}\right\rangle}(s)$ can be written for $s \rightarrow \infty$ as [17]

$$
\widetilde{\left\langle X^{2}\right\rangle}(s)=\sum_{i=0}^{\infty} a_{i} \Gamma\left(\mu_{i}\right) \frac{1}{s^{\mu_{i}}}=2 a_{0} \frac{1}{s^{3}}+6 a_{1} \frac{1}{s^{4}}+24 a_{2} \frac{1}{s^{5}} \cdots .
$$

Substituting equation (17) up to $\mathrm{O}\left(s^{-4}\right)$ in equation (13) yields

$$
\tilde{f}_{\mathrm{I}}(X, s)_{s \rightarrow \infty}=\frac{1}{2 \sqrt{a_{0}}} \exp \left[-\frac{|X|}{\sqrt{a_{0}}}\left(s-\frac{3 a_{1}}{2 a_{0}}\right)\right] .
$$

The inverse Laplace transform of this result reads

$$
f_{\mathrm{I}}(X, t)=\frac{1}{2} \exp \left(\frac{3 a_{1}}{2 a_{0}} t\right) \delta\left(|X|-\sqrt{a_{0}} t\right) .
$$

Not surprisingly, this partial solution corresponds to a deterministic propagation. Note that in order to avoid exponential divergence in time we must have $a_{1}<0$ in the expansion (16).

Having found $\tilde{f}_{\mathrm{I}}(X, s)$ we can now write $\tilde{f}_{\mathrm{II}}(X, s)$ simply as

$$
\tilde{f}_{\mathrm{II}}(X, s)=\tilde{f}(X, s)-\tilde{f}_{\mathrm{I}}(X, s) .
$$

Calculating this difference explicitly we find

$$
\begin{aligned}
\tilde{f}_{\mathrm{II}}(X, s)= & \frac{1}{2}\left\{\sqrt{\frac{2}{\left.s^{3}\left\langle X^{2}\right\rangle(s)\right)}} \exp \left[-|X|\left(\sqrt{\frac{2}{s\left\langle X^{2}\right\rangle(s)}}-\frac{s}{\sqrt{a_{0}}}\right)\right]-\frac{1}{\sqrt{a_{0}}} \exp \left(\frac{3 a_{1}}{2 a_{0}^{3 / 2}}|X|\right)\right\} \\
& \times \exp \left(-\frac{|X|}{\sqrt{a_{0}}} s\right) \equiv \tilde{F}(X, s) \exp \left(-\frac{|X|}{\sqrt{a_{0}}} s\right) .
\end{aligned}
$$

The inverse Laplace transform of equation (21) is given by

$$
f_{\mathrm{II}}(X, t)=F\left(X, t-\frac{|X|}{\sqrt{a_{0}}}\right) H\left(\sqrt{a_{0}} t-|X|\right) .
$$

The importance of this result is that the explicit Heaviside function is taking upon itself the discontinuity in the solution $f_{\mathrm{II}}(X, t)$. The exact value of the function $F\left(X, t-|X| / \sqrt{a_{0}}\right)$ at the point $|X|=\sqrt{a_{0}} t$ can be calculated using the initial value theorem and is given by

$$
F(X, 0)=-\left\{\frac{3}{4} \frac{a_{1}}{a_{0}^{3 / 2}}+\frac{1}{2 \sqrt{a_{0}}}\left[\frac{27}{8}\left(\frac{a_{1}}{a_{0}}\right)^{2}-6 \frac{a_{2}}{a_{0}}\right] t\right\} \exp \left(-\frac{3}{2} \frac{a_{1}}{a_{0}} t\right) .
$$

Summing together the results (19) and (22) in the time domain we get a general solution of the non-Markovian problem with a short-time ballistic behavior, in the form

$$
f(X, t)=\frac{1}{2} \exp \left(\frac{3 a_{1}}{2 a_{0}} t\right) \delta\left(|X|-\sqrt{a_{0}} t\right)+F\left(X, t-\frac{|X|}{\sqrt{a_{0}}}\right) H\left(\sqrt{a_{0}} t-|X|\right) .
$$

This is the main result of the present Letter. The diffusion repartition of the probability distribution function occurs inside the spatial diffusion domain which increases in a deterministic way. The first 


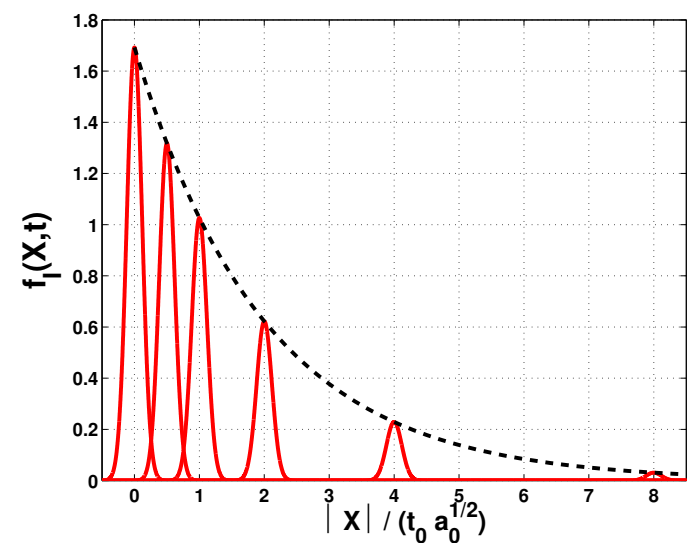

Figure 1. The time evolution of the function $f_{\mathrm{I}}(X, t)$ defined by equation (19) for time intervals $t / t_{0}=0.5,1,2,4,8$ (the time scale $\left.t_{0}=a_{0} /\left(3 a_{1}\right)\right)$. The $\delta$-function is graphically represented by narrow Gaussians.

term in equation (24) corresponds to the propagating $\delta$-function which is inherited from the initial conditions, and it lives at the edge of the ballistically expanding domain. Schematically the time evolution of this term is shown in figure 1, where the $\delta$-function is graphically represented as a narrow Gaussian. The dashed line represents the exponential decay of the integral over the $\delta$ function. The function $F\left(X, t-|X| / \sqrt{a_{0}}\right)$ in the time domain is a continuous function and can be evaluated numerically, for example using the direct integration method [18]. Below we demonstrate this calculation with explicit examples.

\section{Interpolation for all times}

To interpolate equation (2) and (11) we propose the form

$$
\left\langle\Delta X^{2}\right\rangle_{t}=2 D_{\alpha} t_{0}^{\alpha} \frac{\left(t / t_{0}\right)^{2}}{\left(1+\left(t / t_{0}\right)\right)^{2-\alpha}},
$$

where $0 \leqslant \alpha \leqslant 2$. Here $t_{0}$ is the crossover characteristic time, at $t \ll t_{0}$ the law (25) describes the ballistic regime and at $t \gg t_{0}$ the fractional diffusion. Introduce now dimensionless variables $\left\langle\xi^{2}\right\rangle_{\tau}=\left\langle\Delta X^{2}\right\rangle_{t} /\left(2 D_{\alpha} t_{0}^{\alpha}\right)$ and $\tau=t / t_{0}$. With these variables the last equation reads

$$
\left\langle\xi^{2}\right\rangle_{\tau}=\frac{\tau^{2}}{(1+\tau)^{2-\alpha}}
$$

The Taylor expansion of (25) is given by

$$
\left\langle\xi^{2}\right\rangle_{\tau}=\tau^{2}-(2-\alpha) \tau^{3}+\frac{1}{2}(3-\alpha)(2-\alpha) \tau^{4}+\cdots
$$

Substitution of these expansion coefficients into equation (19) yields the first term in the expression for the probability distribution function (24)

$$
f_{\mathrm{I}}(x, t)=\frac{1}{2} \exp \left[-\frac{3(2-\alpha)}{2} \tau\right] \delta(|\xi|-\tau) .
$$

The Laplace transform of equation (26) is

$$
\widetilde{\left\langle X^{2}\right\rangle}(s)=\left(\frac{\alpha}{s}-1\right) \frac{1}{s}+\left[(\alpha-1)\left(\frac{\alpha}{s}-2\right)+s\right] \frac{\mathrm{e}^{s}}{s^{\alpha}} \Gamma(\alpha-1, s),
$$




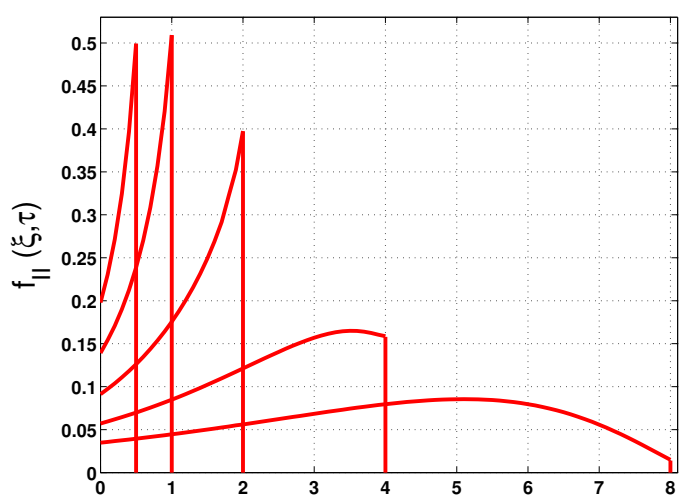

a)

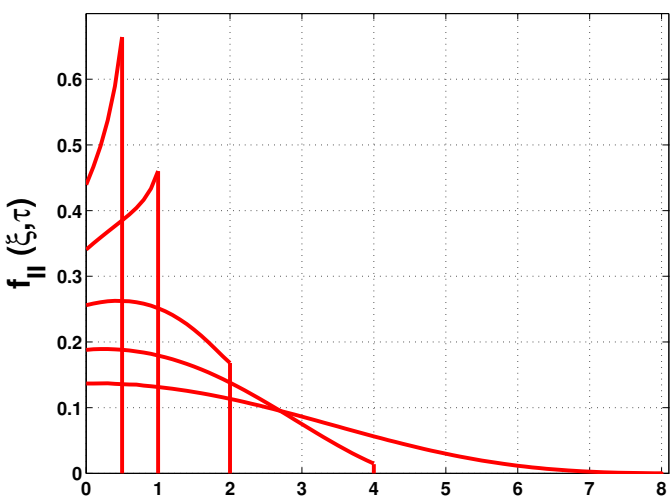

b)

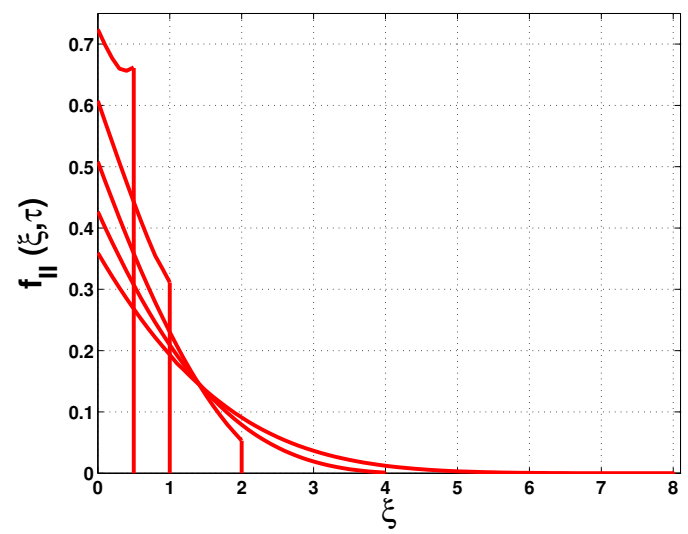

c)

Figure 2. The continuous part of the probability distribution function (22) for different values of the parameter $\alpha$. Superdiffusion $(\alpha=3 / 2)$, case a) ), regular diffusion $(\alpha=1$, case b) ) and subdiffusion $(\alpha=1 / 2$, case $\mathrm{c})$ ). Time intervals from the top to the bottom $\tau=0.5,1,2,4$, 8. The reader should note that the full solution of the problem is the sum of the two solutions shown in this and the previous figure.

where $\Gamma(a, s)$ is the incomplete gamma function. Note that the case $\alpha=2$ is special, since it annuls the exponent in equation (28), leaving as a solution a ballistically propagating $\delta$-function. For all other values of $\alpha<2$ the inverse Laplace transform of the function $\widetilde{F}(x, s)$ which defines the diffusion process inside the expanded spatial domain should be evaluated, in general, numerically.

Results of the calculations following the method of Ref. [18] for the smooth part of the probability distribution function $f_{\mathrm{II}}(x, t)$ for different values of the parameter $\alpha$ are shown in figure 2. The reader should appreciate the tremendous role of memory. For example regular diffusion with $\alpha=1$ results in a Gaussian probability distribution function that is peacefully expanding and flattening as time increases. Here, in the mid panel of figure 2 we see that the ballistic part which is represented by the advancing and reducing $\delta$-function sends backwards the probability that it loses due to the exponential decay seen in figure 1. This "back-diffusion" leads initially to a qualitatively different probability distribution function, with a maximum at the edge of the ballistically expanding domain. At later times the probability distribution function begins to resemble more regular diffusion. The effect strongly depends on $\alpha$ simply due to the appearance of $\alpha$ in the exponent in equation (28).

For long times, the contribution from $f_{\mathrm{I}}(X, t)$ to the general solution tends to zero and the solutions shown in figure 2 agree with the Markovian probability distribution function obtained in the frame of a continuous-time random walk [19]. For the special case $\alpha=0$ equation (29) reads as $\widetilde{\left\langle X^{2}\right\rangle}(s)=1 / s+\left[1-(2+s) \mathrm{e}^{s} E_{1}(s)\right]$, where $E_{1}(s)$ is the exponential integral, and the limiting 
behavior of the general solution from equation (24) $f(X, t)_{t \gg|X| / \sqrt{a_{0}}} \sim F(X, t)$ can be evaluated analytically with the help of the final value theorem $f(X)=1 / \sqrt{2} \exp (-\sqrt{2}|X|)$; this result coincides with the probability distribution function from [19] at the same conditions.

\section{Conclusions}

In summary, we have shown how to deal with diffusion processes that cross-over from a ballistic to a fractional behavior for short and long times, respectively, within the time non-local approach. The general solution (24) demonstrates the effect of the temporal memory in the form of a partition of the probability distribution function inside a spatial domain which increases in a deterministic way. The approach provides a solution that is valid at all times, and in particular is free from the instantaneous action puzzle.

\section{Acknowledgements}

One of the authors (A.Z.) is grateful for the hospitality during his visit to the Department of Chemical Physics, the Weizmann Institute of Science. The work was partially supported by the Program of Fundamental Research of the Division of Physics and Astronomy of the National Academy of Sciences of Ukraine.

\section{References}

1. Hurst H.E., Trans. Am. Soc. Civ. Eng., 1951, 116, 770.

2. Mandelbrot B.B., van Ness J. W., SIAM Rev., 1968, 10, 422-437.

3. Rinn B., Dietriech W., Maass P., Phil. Mag. B, 1998, 77, 1283.

4. Klemm A., Müller H.-P., Kimmich R., Physica A, 1999, 266, 242.

5. Klammler F., Kimmich R., Croat. Chem. Acta, 1992, 65, 455.

6. Fischer E., Kimmich R., Beginn U., Moeller M., Fatkullin N., Phys. Rev. E, 1999, 59, 4079.

7. Porto M., Bunde A., Havlin S., Roman H.E., Phys. Rev. E, 1997, 56, 1667.

8. Cardoso O., Tabeling P., Europhys. Lett, 1988, 7, 225.

9. Weeks E.R., Swinney H.L., Phys. Rev. E, 1998, 57, 4915.

10. Luedtke W.D., Landmann U., Phys. Rev. Lett., 1999, 82, 3835.

11. Richardson L.F., Proc. Roy. Soc., 1926, 110, 709.

12. Bodurka J., Seitter R.-O., Kimmich R., Gutsze A., J. Chem. Phys., 1997, 107, 5621.

13. Schaufler S., Schleich W.P., Yakovlev V.P., Phys. Rev. Lett., 1999, 83, 3162.

14. Metzler R., Klafter J., Phys. Rep., 2000, 339, 1-77.

15. Kenkre V.M., Knox R.S., Phys. Rev. B, 1974, 9, 5279-5290.

16. Sokolov I.M., Phys. Rev. E, 2002, 66, 41101-41105.

17. Van der Pol B., Bremmer H., Operational Calculus, Cambrige, Univ. Press, 1964.

18. Duffy D.G., ACM TOMS, 1993, vol 19, 335-359.

19. Ball R. C., Havlin S., Weiss G. H., J. Phys. A: Math. Gen., 1987, 20, 4055-4059. 


\section{Об’єднаний опис балістичних і дифузійних процесів 3 пам'яттю: точні результати}

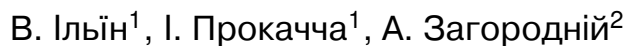

1 Відділ Хімічної Фізики, Інститут Вайсмана, Реховот 76100, Ізраїль

2 Інститут теоретичної фізики ім. М.М. Боголюбова, Київ 03680, Україна

Пропонується об'єднаний опис перехідних дифузійних процесів від балістичної поведінки на малих часах до дробової дифузії на великих часах. 3 цією метою використано немарківське рівняння дифузії. Встановлено явний вигляд часо-нелокального коефіцієнта дифузії, який відповідає різним асимптотикам дифузійного процесу. Знайдено розв'язки такого рівняння і показано, що функція розподілу є неперервною функцією координат і часу в межах області балістичної еволюції. Загальний розв'язок на великих часах узгоджується з результатами, одержаними на основі теорії неперервних в часі випадкових блукань, а на малих часах описує балістичний режим.

Ключові слова: дробова дифузія, ефекти пам'яті, балістичні процеси 